\title{
Role of CaMKK2 in Osteocytes within the Bone Microenvironment
}

\author{
Nick Clough ${ }^{1}$, Justin Williams ${ }^{2}$, Uma Sankar ${ }^{2}$ \\ ${ }^{1}$ Indiana University School of Medicine, ${ }^{2}$ Indiana University School of Medicine, \\ Department of Anatomy and Cell Biology
}

\section{Background and Hypothesis:}

The $\mathrm{Ca}^{+2} /$ calmodulin (CaM)-mediated protein kinase kinase 2 (CaMKK2) is a multi-functional kinase with effects on cell proliferation, differentiation and metabolism. The role of CaMKK2 in bone has been explored with its ablation favoring osteoblasts to osteoclasts and bone mass accrual as observed in Camkk2 $2^{-/-}$mice, or following its inhibition by STO-609. One outstanding question is whether the anabolic effects of CaMKK2 are bone-cell intrinsic. While analyzing mice harboring bone-cell specific deletion of CaMKK2, we observed a high bone mass phenotype when the kinase is deleted from osteocytes, the most abundant cells within the bone. We therefore hypothesized that the loss of CaMKK2 enhances osteocyte differentiation.

\section{Experimental Design or Project Methods:}

We used two osteocyte cell lines MLO-Y4 and MLO-A5, both generated from mice expressing the immortalizing T-antigen, to test our hypothesis. The MLO-A5 line has post-osteoblast/pre-osteocyte characteristics while the MLO-Y4 line has mature osteocyte characteristics. CaMKK2 expression was silenced in MLO-A5 cells using Lentiviruses encoding CaMKK2 short hairpin (sh) RNA constructs. STO-609 was employed to inhibit CaMKK2 in the MLO-Y4 line as it proved resistant to transfection. Immunoblotting was used to verify CaMKK2 silencing/inhibition. Comparisons on cell morphologies were observed using immunofluorescence. As osteocytes are defined by dendritic morphology, the number of dendritic processes were analyzed. Additionally, the differences in the expression of the osteocyte markers SOST, E11 and DMP1 were examined by qRT-PCR.

\section{Results:}

To be finalized.

\section{Conclusion and Potential Impact:}

Overall, our studies will provide more information towards understanding the role of CaMKK2 in bone biology and aid its development as a therapeutic target in the treatment of osteoporosis. 